\title{
SPLITTING IN RELATION ALGEBRAS
}

\author{
H. ANDRÉKA, R. D. MADDUX, AND I. NÉMETI
}

(Communicated by Andreas R. Blass)

\begin{abstract}
We define a way, which we call splitting, of getting new relation algebras from old ones. We characterize those algebras to which splitting can be applied. We show how to split representable relation algebras in order to obtain nonrepresentable ones, and we give many examples.
\end{abstract}

\section{INTRODUCTION}

The method of splitting atoms, originating with L. Henkin, is well known in cylindric algebra theory and is used to obtain nonrepresentable cylindric algebras from representable ones (see [HMT, 3.2.67 and 3.2.69]). In this paper we adapt this method to relation algebras. The conditions for splittability in relation algebras seem to be more complex than in cylindric algebras, where every atom below $\bar{d}(\alpha \times \alpha)$ can be split.

We use the terminology and notation of [Ma1]. In particular, we will deal with the varieties NA, WA, SA, and RA, of nonassociative, weakly associative, semiassociative, and associative relation algebras. These varieties are defined in [Mal] and are obtained from the variety RA of relation algebras by weakening the axiom postulating associativity of relative multiplication. If $\mathfrak{A}$ is any Boolean algebra with additional operations, then At $\mathfrak{A}$ denotes the set of all atoms of the Boolean reduct of $\mathfrak{A}$. Finally, our convention is that; binds more strongly than '. For example, $x ; \check{x} \cdot 0$ ' denotes the term $(x ; \check{x}) \cdot 0$ '.

\section{Definition AND basic PROPERTIES OF SPlitTing}

Definition 1. Let $\mathfrak{A}$ and $\mathfrak{B}$ be atomic NA's. We say that $\mathfrak{A}$ is obtained from $\mathfrak{B}$ by splitting if the following three conditions are met:

(1) $\mathfrak{A} \supseteq \mathfrak{B}$;

(2) every atom $x$ of $\mathfrak{A}$ is contained in an atom $c(x)$ of $\mathfrak{B}$, called the cover of $x ;$ and

Received by the editors October 30,1988 and, in revised form, May 18, 1990.

1980 Mathematics Subject Classification (1985 Revision). Primary 03G15.

Research supported by the Hungarian National Foundation for Scientific Research grant No. 1810. 
(3) for all $x, y \in$ At $\mathfrak{A}$, if $x, y \leq 0$ ' then

$$
x ; y= \begin{cases}c(x) ; c(y) \cdot 0, & \text { if } x \neq \check{y}, \\ c(x) ; c(y) & \text { if } x=\check{y} .\end{cases}
$$

Let $\alpha_{n}$ and $\alpha_{s}$ be functions mapping At $\mathfrak{B}$ to cardinals. We say that $\mathfrak{A}$ is obtained from $\mathfrak{B}$ by splitting along $\alpha_{n}$ and $\alpha_{s}$ if $\mathfrak{A}$ is obtained from $\mathfrak{B}$ by splitting, and for all $x \in$ At $\mathfrak{B}$,

$$
\alpha_{n}(x)=\mid\{y \in \text { At } \mathfrak{A}: y \leq x, y \neq \check{y}\} \mid
$$

and

$$
\alpha_{s}(x)=\mid\{y \in \text { At } \mathfrak{A}: y \leq x, y=\check{y}\} \mid \text {. }
$$

The following lemma says that the result of splitting a complete atomic NA along two functions, if it exists, is unique up to isomorphism. Its proof is straightforward and is therefore omitted.

Lemma 2. Let $\mathfrak{A}, \mathfrak{A}^{\prime}$, and $\mathfrak{B}$ be complete atomic NA's. Let $\alpha_{n}$ and $\alpha_{s}$ be functions mapping At $\mathfrak{B}$ to cardinals. If $\mathfrak{A}$ and $\mathfrak{A}^{\prime}$ are obtained from $\mathfrak{B}$ by splitting along $\alpha_{n}$ and $\alpha_{s}$, then $\mathfrak{A}$ and $\mathfrak{A}^{\prime}$ are isomorphic by an isomorphism which leaves $\mathfrak{B}$ fixed.

The next theorem characterizes those functions along which an atomic NA, WA, SA, or RA can be split so as to obtain an algebra in the same class. Intuitively, in NA we have only the obvious conditions, namely, (a) in Theorem 3; in WA, the additional condition is that we cannot split "nondiscrete" identity elements; in SA, the additional condition is that we cannot split nondiscrete functional elements; and in RA we have a slightly stronger condition.

If $\mathfrak{A} \in N A$, then Fna is the set of functional elements of $\mathfrak{A}$. Thus Fn $\mathfrak{A}=$ $\left\{x \in A: \check{x} ; x \leq 1^{\prime}\right\}$.

Theorem 3. Let $\mathfrak{B}$ be an atomic NA. Let $\alpha_{n}, \alpha_{s}$ be functions mapping At $\mathfrak{B}$ to cardinals, and let $\alpha(x)=\alpha_{n}(x)+\alpha_{s}(x)$. Consider conditions (a)-(d):

(a) For all $x \in$ At $\mathfrak{B}, \alpha(x) \geq 1, \alpha_{n}(x)=\alpha_{n}(\check{x})$, if $x \leq 1$ ', then $\alpha_{n}(x)=0$; if $x=\check{x}$, then $\alpha_{n}(x)=2 \kappa$ for some $\kappa$; and if $x \neq \check{x}$, then $\alpha_{s}(x)=0$.

(b) For all $x \in$ At $\mathfrak{B}$, if $\alpha(x)>1$ and $0^{\prime} ; x \neq 0$, then $x \leq 0^{\prime}$.

(c) For all $x \in$ At $\mathfrak{B}$, if $\alpha(x)>1$ and $0^{\prime} ; x \neq 0$, then $x \notin$ Fn $\mathfrak{A}$.

(d) For all $x, y \in$ At $\mathfrak{B}$, if $\alpha(x)>1, y ; x \neq 0$, and $y \leq 0$, then $y \leq$ $y ;\left(x ; \check{x} \cdot 0^{\prime}\right)$.

Then

(i) There is an atomic $\mathfrak{A} \in$ NA obtained from $\mathfrak{B}$ by splitting along $\alpha_{n}$ and $\alpha_{s}$ iff (a) holds.

Now suppose $\mathfrak{A} \in \mathrm{NA}, \mathfrak{A}$ is atomic, and $\mathfrak{A}$ obtained from $\mathfrak{B}$ by splitting along $\alpha_{n}$ and $\alpha_{s}$. Then

(ii) $\mathfrak{A} \in \mathrm{WA}$ iff $\mathfrak{B} \in \mathrm{WA}$ and (b) holds, 
(iii) $\mathfrak{A} \in \mathrm{SA}$ iff $\mathfrak{B} \in \mathrm{SA}$ and (c) holds,

(iv) $\mathfrak{A} \in \mathrm{RA}$ iff $\mathfrak{B} \in \mathrm{RA}$ and (d) holds.

Proof. First we prove the "only if" parts. Assume $\mathfrak{A}$ and $\mathfrak{B}$ are atomic NA's and $\mathfrak{A}$ is obtained from $\mathfrak{B}$ by splitting along $\alpha_{n}$ and $\alpha_{s}$. Then (a) holds by the elementary laws governing in NA's (namely, by [Ma1, 1.13(16)(12)(8)]). Clearly, if $\mathfrak{A} \in W A$, then $\mathfrak{B} \in W A$ by Definition $1(1)$, and similarly for $S A$ and RA. Next we show that (b), (c), or (d) holds whenever $\mathfrak{A}$ is in WA, SA, or RA, respectively.

Assume $\mathfrak{A} \in \mathrm{WA}, 1^{\prime} \geq x \in \mathrm{At} \mathfrak{B}$, and $0^{\prime} ; x \neq 0$. Then there is some $y \in$ At $\mathfrak{B}$ such that $y \leq 0$ ' and $y ; x \neq 0$. Then $y ; x=y$ since $x \leq 1^{\prime}$ and $y \in$ At $\mathfrak{B}$. Recall that $y^{r}=\check{y} ; y \cdot 1^{\prime}$ and $y^{\mathrm{d}}=y ; \check{y} \cdot 1$ ' (see [Ma1, 5.10]). Then $y^{r}=x$ by $[\mathrm{Ma} 2,5.12(3)]$. Suppose $y \geq y_{0} \in$ At $\mathfrak{A}$. Then $y_{0}^{r}=x$ by Definition 1(3); hence, $x \in A t \mathfrak{A}$ since $y_{0} \in A t \mathfrak{A}$, by [Ma2, 5.12(1)]. Therefore $\alpha(x)=1$.

Assume $\mathfrak{A} \in \mathrm{SA}, x \in$ At $\mathfrak{B}, \alpha(x)>1$, and 0 '; $x \neq 0$. Then $x \leq 0$ ' by (b), and there are $x_{0}, x_{1} \in$ At $\mathfrak{A}$ such that $x_{0} \neq x_{1}$ and $c\left(x_{0}\right)=c\left(x_{1}\right)=x$. Then $x_{0}^{\mathrm{d}}=x_{1}^{\mathrm{d}}=x^{\mathrm{d}}$ by Definition $1(3)$, so $x_{1}=x_{1}^{\mathrm{d}} ; x_{1}=x_{0}^{\mathrm{d}} ; x_{1}=\left(x_{0} ; \check{x}_{0} \cdot 1\right.$ ') $; x_{1} \leq$ $\left(1 ; \check{x}_{0}\right) ; x_{1}=1 ;\left(\check{x}_{0} ; x_{1}\right)$ by [Ma1, 5.11(3)], and the semiassociative law. Thus $\check{x}_{0} ; x_{1} \neq 0$. We have $\check{x}_{0} ; x_{1}=\check{x} ; x \cdot 0$ ' by Definition $1(3)$, so $\check{x} ; x \not 11$ '.

To prove that (d) holds when $\mathfrak{A} \in \mathrm{RA}$, we will need (*) below. We note that (*) is true if $\mathfrak{A} \in N A$.

If $x \in \mathrm{At} \mathfrak{B}, y \in \mathrm{At} \mathfrak{A}$, and $x, y \leq 0^{\prime}$, then $x ; y=x ; c(y)$.

To prove $(*)$, assume $x \in$ At $\mathfrak{B}, y \in$ At $\mathfrak{A}$, and $x, y \leq 0$. Clearly $x ; y \leq$ $x ; c(y)$. For the opposite direction, first note that if $x=c(\check{y})$, then $x ; c(y)=$ $c(\check{y}) ; c(y)=\check{y} ; y \leq x ; y$. Assume $x \neq c(\check{y})$. Choose $z \in$ At $\mathfrak{A}$ so that

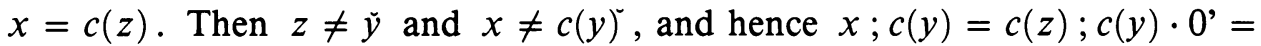
$z ; y \leq x ; y$. (*) has been proved.

Assume $\mathfrak{A} \in \mathrm{RA}, x, y \in$ At $\mathfrak{B}, y ; x \neq 0, y \leq 0$, and $\alpha(x)>1$. Then $x \leq 0$ ' by (b). Let $x_{0}, x_{1} \in$ At $\mathfrak{A}$ be such that $x_{0} \neq x_{1}$ and $c\left(x_{0}\right)=c\left(x_{1}\right)=x$. Then $x_{0} ; \check{x}_{1}=x ; \check{x} \cdot 0$ ' by Definition $1(3)$, and $0 \neq y ; x=y ; c\left(x_{0}\right) \cdot y ; c\left(x_{1}\right)=$ $y ; x_{0} \cdot y ; x_{1}$ by $(*)$, so $0 \neq y \cdot\left(y ; x_{0}\right) ; \check{x}_{1}=y \cdot y ;\left(x_{0} ; \check{x}_{1}\right)=y \cdot y ;\left(x ; \check{x} \cdot 0^{\prime}\right)$ by the associative law, and therefore $y \leq y ;(x ; \check{x} \cdot 0$ ').

We now turn to proving the "if" parts. Let $\mathfrak{B}, \alpha_{n}, \alpha_{s}$, and $\alpha$ be as in the statement of the theorem, and assume (a) holds. We may assume that $\mathfrak{B}$ is complete. We will construct a complete atomic $\mathfrak{A} \in N A$ that is obtained from $\mathfrak{B}$ by splitting along $\alpha_{n}$ and $\alpha_{s}$. Then we prove that $\mathfrak{A} \in W A$ if (b) holds and $\mathfrak{B} \in W A$, that $\mathfrak{A} \in S A$ if (c) holds and $\mathfrak{B} \in S A$, and that $\mathfrak{A} \in R A$ if (d) holds and $\mathfrak{B} \in \mathrm{RA}$.

First we will construct a copy of $\mathfrak{A}$, namely $\mathfrak{A}^{\prime}$. It will be the complex algebra of a certain relational structure $\langle U, C, f, I\rangle$, where $C \subseteq U \times U \times U, f$ is an involution of $U$, and $I \subseteq U$. (See [Ma2, Definition 2.1].) For every $x \in$ At $\mathfrak{B}$, let $S_{n}(x)$ and $S_{s}(x)$ be sets such that $\left|S_{n}(x)\right|=\alpha_{n}(x),\left|S_{s}(x)\right|=\alpha_{s}(x)$, and, 
for all $i, j \in\{n, s\}$ and all $x, y \in$ At $\mathfrak{B}$, we have $S_{i}(x) \cap S_{j}(y)=\varnothing$ if $i \neq j$ or $x \neq y$. Set $U=\bigcup\left\{S_{n}(x) \cup S_{s}(x): x \in\right.$ At $\left.\mathfrak{B}\right\}$. Let $f$ be a function mapping $U$ to $U$ such that for all $y \in U$ we have $f f(y)=y, f(y)=y$ iff $y \in S_{s}(x)$ for some $x \in$ At $\mathfrak{B}$, and for all $x \in$ At $\mathfrak{B}$, if $y \in S_{n}(x)$, then $f(y) \in S_{n}(\check{x})$. Such a function $f$ exists by (a). Let $c$ be the function mapping $U$ to At $B$ defined by $c(y)=x$ if $y \in S_{n}(x) \cup S_{s}(x)$. For all $x, y, z \in U$, let

$$
\begin{aligned}
& {[x, y, z]=\{\langle x, y, z\rangle,\langle f x, z, y\rangle,} \\
&\langle y, f z, f x\rangle,\langle f y, f x, f z\rangle,\langle f z, x, f y\rangle,\langle z, f y, x\rangle\} .
\end{aligned}
$$

Let

$$
\begin{aligned}
C= & \bigcup\left\{[x, y, z]: c(x) ; c(y) \geq c(z) \text { and } c(x), c(y), c(z) \leq 0^{\prime}\right\} \\
& \cup \bigcup\{[x, y, y]: c(x) ; c(y) \geq c(y), c(x) \leq 1 \text { ', and } \\
& \text { either } c(y) \leq 0 \text { 'or } y=x\},
\end{aligned}
$$

and let $I=\left\{x: x \in U\right.$ and $\left.c(x) \leq 1^{\prime}\right\}$. Set $\mathfrak{A}^{\prime}=\mathfrak{C m}\langle U, C, f, I\rangle$.

It follows directly from the definitions and [Ma1, 2.2(2)] that $\mathfrak{A}^{\prime} \in N A$. For every $b \in B$ let $h(b)=\{y: y \in U$ and $c(y) \leq b\}$. It is easy to check that $h$ is an embedding of $\mathfrak{B}$ into $\mathfrak{A}^{\prime}$, and that $\mathfrak{A}^{\prime}$ is obtained from the image of $\mathfrak{B}$ by splitting along $\alpha_{n}$ and $\alpha_{s}$. We may then obtain $\mathfrak{A}$ by replacing the image of $\mathfrak{B}$ in $\mathfrak{A}^{\prime}$ with $\mathfrak{B}$ itself.

Now assume (b) and $\mathfrak{B} \in W A$. To show $\mathfrak{A}^{\prime} \in W A$ it suffices to check condition [Ma1, 2.2(d)]. Assume $w \in I$ and $\langle w, x, x\rangle \in C$ and $\langle x, y, z\rangle \in$ $C$. Then $c(w) ; c(x) \geq c(x)$ and $c(x) ; c(y) \geq c(z)$ in $\mathfrak{B}$; hence, $c(w) ; c(z) \geq$ $c(z)$, since $\mathfrak{B} \in W A$. If $c(z) \leq 0$, then $\langle w, z, z\rangle \in C$, and we are done. Assume $c(z) \leq 1^{\prime}$. Then $c(w)=c(z)$. If $c(x) \leq 1^{\prime}$, then $x=y=z$, since $\langle x, y, z\rangle \in C$. Hence $\langle w, z, z\rangle \in C$, and we are done. If $c(x) \leq 0^{\prime}$, then $c(x)^{\circ} \leq 0^{\prime}$ and $0 \neq c(x)^{\circ} \leq c(x)^{\circ} ; c(w)$, so $\alpha(c(w))=1$ by (b). Hence $z=w$, since $c(z)=c(w)$, so $\langle w, z, z\rangle \in C$, and we are done.

Now assume (c) and $\mathfrak{B} \in \mathrm{SA}$. We want to check [Ma1, 2.2(e)]. Let $\langle v, w, x\rangle,\langle x, y, z\rangle \in C$. We have to find some $u \in U$ for which $\langle v, u, z\rangle \in$ $C$. We get (b) from (c), since for every $x \in$ At $\mathfrak{A}$, either $x \leq 1^{\prime}$ or $x \leq 0^{\prime}$, but if $x \leq 1^{\prime}$ then $x \in \mathrm{Fn} \mathfrak{A}$. From what has been proved so far, we have $\mathfrak{A}^{\prime} \in \mathrm{WA}$, and [Ma1, 2.2(d)] holds. If $v \in I$, then $w=x$ by the definition of $C$, so we have $\langle v, x, x\rangle,\langle x, y, z\rangle \in C$, and hence $\langle v, z, z\rangle \in C$ by $2.2(\mathrm{~d})$, so $u=z$ will do. From $\langle v, w, x\rangle,\langle x, y, z\rangle \in C$, we get $\langle z, f(y), x\rangle,\langle x, f(w), v\rangle \in$ $C$. If $z \in I$, then $f(y)=x$, so $\langle z, x, x\rangle,\langle x, f(w), v\rangle \in C$; hence, $\langle z, v, v\rangle \in C$ by $2.2(\mathrm{~d})$, and finally $\langle v, f(v), z\rangle \in C$, so $u=f(v)$ will do. Therefore we may assume $v \notin I$ and $z \notin I$. Since $\langle v, w, x\rangle,\langle x, y, z\rangle \in$ $C$, we have $c(v) ; c(w) \geq c(x)$ and $c(x) ; c(y) \geq c(z)$. Since $\mathfrak{B} \in \mathrm{SA}$, $c(z) \leq(c(v) ; c(w)) ; c(y) \leq(c(v) ; 1) ; 1=c(v) ; 1$, so there is some $c(u) \in$ At $\mathfrak{B}$ such that $c(v) ; c(u) \geq c(z)$. If $c(u) \leq 0$, then $\langle v, u, z\rangle \in C$, and we are done. Assume $c(u) \leq 1$ '. Then $c(v)=c(z)$. If $v=z$, then $\langle v, u, z\rangle=\langle v, u, v\rangle \in[u, f(v), f(v)] \subseteq C$. If $v \neq z$, then $\alpha(c(v))>1$, 
and $c(u) \leq c(z)^{r} ; c(v) \leq 0^{\prime} ; c(v)$, so $c(v) \notin \mathrm{Fn} \mathfrak{A}$ by (c). Hence there is some $c\left(u^{\prime}\right) \in$ At $\mathfrak{B}$ such that $c\left(u^{\prime}\right) \leq 0$ and $c(v) ; c\left(u^{\prime}\right) \geq c(z)$. Now $\left\langle v, u^{\prime}, z\right\rangle \in C$, and we are done.

Assume (d) and $\mathfrak{B} \in \mathrm{RA}$. To check [Ma1, 2.2(f)], let $\langle v, w, x\rangle,\langle x, y, z\rangle$ $\in C$. We have to find some $u \in U$ for which $\langle v, u, z\rangle,\langle w, y, u\rangle \in C$. First note that (b) holds, since clearly (d) implies (c), so $\mathfrak{A}^{\prime} \in W A$ and 2.2(d) holds. If $v, w, y$, or $z$ is in $I$ then let $u$ be $z, y, w$ or $f(v)$, respectively. In each of these four cases it is easy to show, by arguments similar to those in the previous paragraph, that $\langle v, u, z\rangle,\langle w, y, u\rangle \in C$, using the fact that 2.2 (d) holds. Therefore assume $v, w, y, z \notin I$, and hence $c(v), c(w), c(y), c(z) \leq 0$ '. Because $\mathfrak{B} \in \mathrm{RA}$, there is some $c(u) \in$ At $\mathfrak{B}$ such that $c(v) ; c(u) \geq c(z)$ and $c(w) ; c(y) \geq c(u)$. If $c(u) \leq 0$, then we are done. Assume $c(u) \leq$ 1'. Then $c(v)=c(z)$ and $c(w)=c(y)^{r}$. If $v=z$ and $w=f(y)$, then $\langle v, u, z\rangle,\langle w, y, u\rangle \in C$ and we are done. Assume $v \neq z$. (The case $w \neq$ $f(y)$ is completely analogous, and therefore we omit it.) Then $\alpha(c(v))>1$. By $\langle v, w, x\rangle \in C$ and (d), we have $c(y) \leq c(w)^{\prime} ;\left(c(v)^{r} ; c(z) \cdot 0^{\prime}\right)$, so there is some $c\left(u^{\prime}\right) \in$ At $\mathfrak{B}$ such that $c(y) \leq c(w)^{\prime} ; c\left(u^{\prime}\right)$ and $c\left(u^{\prime}\right) \leq c(v)^{\circ} ; c(z) \cdot 0^{\prime}$. Then $c\left(u^{\prime}\right) \leq c(w) ; c(y)$ and $c(z) \leq c(v) ; c\left(u^{\prime}\right)$, so $\left\langle v, u^{\prime}, z\right\rangle,\left\langle w, y, u^{\prime}\right\rangle \in C$ and we are done.

We now turn to investigating the condition of splittability in RA's.

Definition 4. Suppose $\mathfrak{A} \in \mathrm{RA}, \mathfrak{A}$ is atomic, and $x \in A \mathrm{~A} \mathfrak{A}$. We say that $x$ is splittable in $\mathfrak{A}$ if $x \leq 0^{\prime}$ and $y \leq y ;\left(x ; \check{x} \cdot 0^{\prime}\right)$ whenever $0^{\prime} \geq y \in$ At $\mathfrak{A}$ and $y ; x \neq 0$, and $y \leq\left(\check{x} ; x \cdot 0^{\prime}\right) ; y$ whenever $0^{\prime} \geq y \in$ At $\mathfrak{A}$ and $x ; y \neq 0$. We say that $\mathfrak{A}$ is splittable iff $\mathfrak{A}$ has a splittable atom.

By Theorem $3, x$ is splittable in $\mathfrak{A}$ iff there is some $\mathfrak{B} \in \mathrm{RA}$ obtained from $\mathfrak{A}$ by splitting such that $x \notin$ At $\mathfrak{B}$. Note that if $x$ is splittable then there is no $y \in$ Fn $A \cap A t \mathfrak{A}$ such that $y \leq 0^{\prime}$ and either $y^{r}=x^{\mathrm{d}}$ or $y^{\mathrm{d}}=x^{r}$. The following lemma says that the converse holds if $x=x ; 1 ; x$.

Lemma 5. Let $\mathfrak{A} \in \mathrm{NA}, x \in$ At $\mathfrak{A}$. Consider statements (1)-(4):

(1) for every $y \in \operatorname{At} \mathfrak{A}$, if $y ; x \neq 0$ and $y \leq 0^{\prime}$, then $y \leq y ;\left(x ; \check{x} \cdot 0^{\prime}\right)$;

(2) for every $y \in \mathrm{Fn} \mathfrak{A} \cap \mathrm{At} \mathfrak{A},\left(0^{\prime} \cdot y\right) ; x=0$;

(3) for every $y \in \operatorname{Fn} \mathfrak{A} \cap \mathrm{A} \pm \mathfrak{A}$, if $y \leq 0^{\prime}$ then $y^{r} \neq x^{\mathrm{d}}$; and

(4) for every $y \in \mathrm{Fn} \mathfrak{A} \cap \mathrm{At} \mathfrak{A}$, if $y^{\mathrm{r}}=x^{\mathrm{d}}$, then $y=x^{\mathrm{d}}$.

Then (1) implies (2), (3), and (4). If $\mathfrak{A} \in \mathrm{SA}$ and $x=x ; 1 ; x$, then (1)-(4) are all equivalent.

Proof. It is easy to show that (1) implies (2) in every NA. Assume $\mathfrak{A} \in \mathrm{SA}$ and $x=x ; 1 ; x$. Assume that (2) holds. Let $y \in$ At $\mathfrak{A}, y ; x \neq 0$, and $y \leq 0$ '. We want to show that $y \leq y ;\left(x ; \check{x} \cdot 0^{\prime}\right)$. We have $y \notin \mathrm{Fn} \mathfrak{A}$ by (2), so $y \leq y ; 0$, since $y \in$ At $\mathfrak{A}$. From $y \in$ At $\mathfrak{A}$ and $y ; x \neq 0$ we get $y \leq 1 ; \check{x}$ and $\check{y} \leq x ; 1$. Using these equations, $\mathfrak{A} \in \mathrm{SA}$, and $x=x ; 1 ; x$, we get

$$
\check{y} ; y \leq \check{y} ; 1 \cdot 1 ; y \leq x ; 1 ; 1 \cdot 1 ;(1 ; \check{x})=x ; 1 \cdot 1 ; \check{x} \leq(x ; 1 ; x) ; \check{x}=x ; \check{x},
$$


so $y=y \cdot y ; 0^{\prime} \leq y ;\left(\check{y} ; y \cdot 0^{\prime}\right) \leq y ;\left(x ; \check{x} \cdot 0^{\prime}\right)$, as desired.

It is easy to see that if $\mathfrak{A} \in \mathrm{SA}$ then (2)-(4) are equivalent by the elementary laws governing $x^{d}$ and $x^{r}$ [Ma1, 5.11 and 5.12].

\section{EXAMPLES}

Examples 1,2, and 3 show that the condition $x=x ; 1 ; x$ cannot be omitted in Lemma 5. Examples 4, 5, and 6 show how to split atoms in order to obtain nonrepresentable RA's from representable ones.

Example 1. Let $\mathfrak{A}$ be the following RA: At $\mathfrak{A}=\{1, a, b\}, \check{a}=a, \check{b}=b$, $a ; a=b+1^{\prime}, b ; b=a+1^{\prime}$, and $a ; b=b ; a=a+b$. It is fairly easy to prove that $\mathfrak{A}$ has only one representation, the one given in Figure 1. (More precisely, $\mathfrak{A}$ is embeddable in $\mathfrak{R} U$, the algebra of all relations on $U$, just in the case that $|U|=5$ and the relations correlated with $a$ and $b$ look like those in Figure 1.)

Now $\mathfrak{A}$ is not splittable, because $a \leq z a ;\left(b ; \check{b} \cdot 0^{\prime}\right)$ and $b \leq b ;\left(a ; \check{a} \cdot 0^{\prime}\right)$.

Example 2. Nonminimal RA's in which all the atoms are equivalence elements are not splittable, because of the following: Suppose $\mathfrak{A} \in \mathrm{RA}, \mathfrak{A}$ is atomic, $\mid$ At $\mathfrak{A} \mid \geq 3$, and for every $a \in$ At $\mathfrak{A}, \check{a}=a$ and $a ; a=a+1$ '. (It follows that $1^{\prime} \in \operatorname{At} \mathfrak{A}$.) Let $0^{\prime} \geq a \in$ At $\mathfrak{A}$. Then there is some $b \in$ At $\mathfrak{A}$ such that $b \leq 0$ ' and $a \neq b$. Now $a ; \check{a} \cdot 0^{\prime}=a$, but $b \leq b ; a$, showing that $a$ is not splittable. Examples of such algebras are the Lyndon algebras (see [L] or [J]). In a Lyndon algebra, if $a$ and $b$ are atoms distinct from each other and from $1^{\prime}$, then $\check{a}=a, a ; a=a+1^{\prime}$, and $a ; b=\left(a+b+1^{\prime}\right)^{-}$.

Example 3. We now examine the three minimal RA's. We will see that two of them are splittable and that all the algebras obtained from them by splitting are representable.

Let $\mathfrak{A}$ be a minimal RA. Then At $\mathfrak{A} \subseteq\left\{1^{\prime}, 0^{\prime}\right\}$ and $0^{\prime} ; 0^{\prime} \in\left\{0,1^{\prime}, 1\right\}$. If $0^{\prime}=0$, then $1^{\prime}=1$. Hence $\mathfrak{A}$ is splittable. The algebras obtained from $\mathfrak{A}$ by splitting are all Boolean relation algebras, and hence are representable. Assume $0^{\prime} ; 0^{\prime}=1$ '. Then $0^{\prime} \in$ Fn $\mathfrak{A}$; hence, 1' and $0^{\prime}$ are not splittable in $\mathfrak{A}$ since

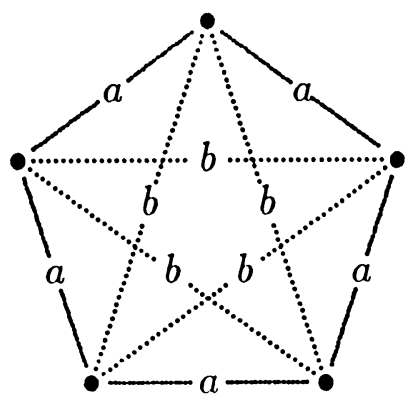

FIGURE 1. Representation of $\mathfrak{A}$ 

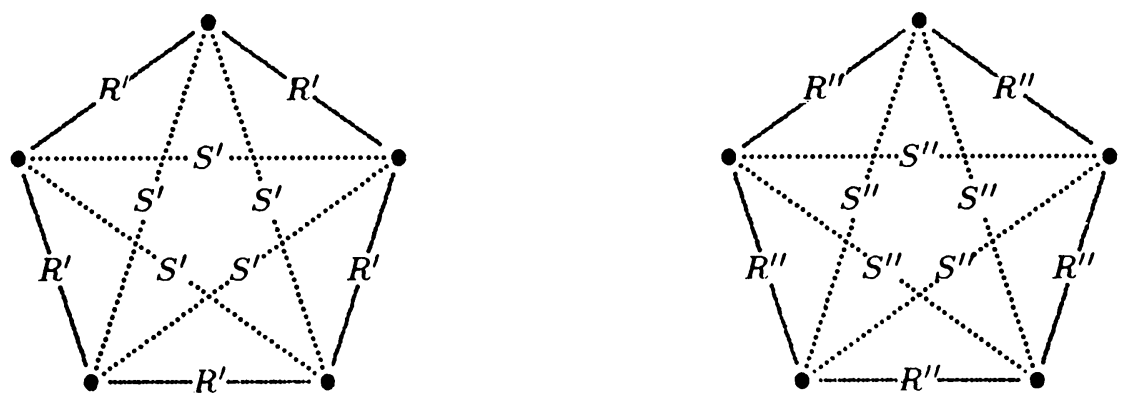

FIGURE 2

$0^{\prime} ; 0^{\prime} \neq 0$ and $0^{\prime} ; 1^{\prime} \neq 0$. Thus $\mathfrak{A}$ is not splittable. Assume $0^{\prime} ; 0^{\prime}=1$. Then 0 ' is splittable in $\mathfrak{A}$ and the algebras $\mathfrak{B}$ obtained from $\mathfrak{A}$ by splitting are the following: At $\mathfrak{B}=\left\{1^{\prime}\right\} \cup S \cup N, \check{a}=a$ for all $a \in S, \check{a} \neq a$ for all $a \in N$, and $a ; a=1, a ; b=0$ ' for all $a, b \in S \cup N$ with $a \neq b$. These algebras are all representable by [Ma1, 5.19]. In the case $N=\varnothing$, these algebras are called $\mathfrak{E}_{n}(\{1,2,3\})$ in [Ma1, 2.4], where $n=\mid$ At $\mathfrak{B} \mid$.

Now we use splitting to obtain nonrepresentable RA's from representable ones.

Example 4. Let $\mathfrak{A}$ be the following proper relation algebra: Set $U=U^{\prime} \cup U^{\prime \prime}$, where $U^{\prime} \cap U^{\prime \prime}=\varnothing$ and $\left|U^{\prime}\right|=\left|U^{\prime \prime}\right|=5$. Let $R^{\prime}, S^{\prime}, R^{\prime \prime}, S^{\prime \prime}$ be the relations shown in Figure 2, and let $\mathfrak{A}$ be the proper relation algebra with atoms $\left\{\operatorname{Id}_{U^{\prime}}, R^{\prime}, S^{\prime}, \operatorname{Id}_{U^{\prime \prime}}, R^{\prime \prime}, S^{\prime \prime}, U^{\prime} \times U^{\prime \prime}, U^{\prime \prime} \times U^{\prime}\right\}$.

Now by Lemma $5, U^{\prime} \times U^{\prime \prime}$ is splittable in $\mathfrak{A}$. Let $\mathfrak{B}$ be the RA obtained from $\mathfrak{A}$ by splitting $U^{\prime} \times U^{\prime \prime}$ into at least six atoms. Then $\mathfrak{B}$ is not representable, because the only representations of $\operatorname{Id}_{U^{\prime}}, R^{\prime}, S^{\prime}$ and $\operatorname{Id}_{U^{\prime \prime}}, R^{\prime \prime}, S^{\prime \prime}$ are the ones shown in Figure 2, and clearly there cannot be six disjoint relations below $U^{\prime} \times U^{\prime \prime}$ with domain $U^{\prime}$.

It is also true that splitting $U^{\prime} \times U^{\prime \prime}$ into two parts already yields a nonrepresentable RA (with ten atoms). When showing this in Example 4a below, we modify Example 4 so that we obtain a symmetric integral nonrepresentable $\mathfrak{B} \in \mathrm{RA}$ with five atoms.

Example 4a. Let everything be as in Example 4. Let $R=R^{\prime}+R^{\prime \prime}, S=S^{\prime}+S^{\prime \prime}$, and $D=\left(U^{\prime} \times U^{\prime \prime}\right)+\left(U^{\prime \prime} \times U^{\prime}\right)$. Let $\mathfrak{A}^{\prime}$ be the proper relation algebra with atoms $\operatorname{Id}_{U}, R, S$, and $D$. It can be checked that $D ; \check{D} \cdot 0^{\prime}=\check{D} ; D \cdot 0^{\prime}=R+S$, and $y \leq y ;(R+S)$ for all $y \in\{R, S, D\}$, so $D$ is splittable in $\mathfrak{A}^{\prime}$. Let $\mathfrak{B}^{\prime} \in \mathrm{RA}$ be obtained from $\mathfrak{A}^{\prime}$ by splitting $D$ into two symmetric parts, say into $D^{\prime}$ and $D^{\prime \prime}$. Then $\mathfrak{B}^{\prime}$ has five atoms, $\operatorname{Id}_{U}, R, S, D^{\prime}, D^{\prime \prime}$. Assume $\mathfrak{B}^{\prime}$ is representable. It can be shown that $\mathfrak{A}^{\prime}$ has only one representation (up to isomorphisms of the ten-element set $U$ ). Therefore we may assume that the representation of $\mathfrak{B}^{\prime}$ is the same as that of $\mathfrak{A}^{\prime}$ and $D=D^{\prime}+D^{\prime \prime}$. Let $u \in U$ be arbitrary. Let $D^{\prime}(u)=\left\{v \in U^{\prime \prime}:\langle u, v\rangle \in D^{\prime}\right\}$ and $D^{\prime \prime}(u)=\left\{v \in U^{\prime \prime}:\langle u, v\rangle \in D^{\prime \prime}\right\}$. Then 
$D^{\prime}(u) \cap D^{\prime \prime}(u)=\varnothing$ and $\left|D^{\prime}(u) \cup D^{\prime \prime}(u)\right|=5$. Now, since $D^{\prime} \leq D=D^{\prime} ; R=$ $D^{\prime} ; S$ and $1^{\prime} \cdot(R+S)=0$, we have $\left|D^{\prime}(u)\right| \geq 3$, and similarly $\left|D^{\prime \prime}(u)\right| \geq 3$, since $D^{\prime \prime} \leq D^{\prime \prime} ; R=D^{\prime \prime} ; S$. This contradicts $\left|D^{\prime}(u) \cup D^{\prime \prime}(u)\right|=5$. Thus $\mathfrak{B}^{\prime}$ is a nonrepresentable symmetric integral RA with five atoms.

The essence of Example 4 was that we had an atom $U^{\prime} \times U^{\prime \prime}$ such that the structure of the algebra forced $U^{\prime}$ to be small in any representation. We then split $U^{\prime} \times U^{\prime \prime}$ into too many parts. Now we use the same idea to obtain a series of nonrepresentable RA's.

Example 5. Let $p=2 n+1$ be any prime number. We will construct a nonrepresentable $\mathfrak{B}_{n} \in \mathrm{RA}$. We start with a proper relation algebra $\mathfrak{A}_{n}^{\prime}$ on $p=$ $\{0,1, \ldots, p-1\}$. In the following, + and - are understood modulo $p$. For $i \in p$, define

$$
R_{i}=\{\langle n, m\rangle:|n-m| \equiv i(\bmod p), n, m \in p\} .
$$

This gives us a proper relation algebra $\mathfrak{A}_{n}^{\prime}$ with atoms $\left\{R_{0}, R_{1}, \ldots, R_{n}\right\}$. In $\mathfrak{A}^{\prime}$ we have $R_{0}=\mathrm{Id}_{p}=1$ ', and if $i<j \leq n$, then $R_{i}=\check{R}_{i}, R_{i} \cdot R_{j}=0$, $1=p \times p=R_{0} \cup \cdots \cup R_{n}$, and $R_{i} ; R_{j}=R_{i+j} \cup R_{i-j}$.

Let $\mathfrak{A}_{n}$ be the proper relation algebra obtained from $\mathfrak{A}_{n}^{\prime}$ by putting two disjoint copies of $\mathfrak{A}_{n}^{\prime}$ together as we did in Example 4; set $U=U^{\prime} \cup U^{\prime \prime}$ where $U^{\prime} \cap U^{\prime \prime}=\varnothing$ and $\left|U^{\prime}\right|=\left|U^{\prime \prime}\right|=p$. Let $R_{1}^{\prime}, \ldots, R_{n}^{\prime}$ and $R_{1}^{\prime \prime}, \ldots, R_{n}^{\prime \prime}$ be the corresponding relations on $U^{\prime}$ and $U^{\prime \prime}$, respectively. Then At $\mathfrak{A}_{n}=$ $\left\{\operatorname{Id}_{U^{\prime}}, R_{1}^{\prime}, \ldots, R_{n}^{\prime}, \operatorname{Id}_{U^{\prime \prime}}, R_{1}^{\prime \prime}, \ldots, R_{n}^{\prime \prime}, U^{\prime} \times U^{\prime \prime}, U^{\prime \prime} \times U^{\prime}\right\}$. Let $\mathfrak{B}_{n}$ be the algebra obtained from $\mathfrak{A}_{n}$ by splitting $U^{\prime} \times U^{\prime \prime}$ into two atoms. By a slight generalization of the argument in Example $4 a$, we see that $\mathfrak{B}_{n}$ is not representable. First, it is easy to show that $\mathfrak{A}_{n}^{\prime}$ has just one representation on a $p$-element set, and hence that $\mathfrak{A}_{n}$ has just one representation on a $2 p$-element set, namely the one used to describe $\mathfrak{A}_{n}$. But $U^{\prime} \times U^{\prime \prime}=D+D^{\prime}$ in $\mathfrak{A}_{n}$, so for any $u \in U^{\prime}$ the images $D(u)$ and $D^{\prime}(u)$ are disjoint subsets of $U^{\prime \prime}$, each of which contains at least $n+1$ elements (since $D=D ; R_{i}$ and $D^{\prime}=D^{\prime} ; R_{i}$ for $i=0, \ldots, n-1)$, contradicting $\left|U^{\prime \prime}\right|=p$.

Example 6. The algebras $\mathfrak{E}_{n}(\{2,3\})$ (see $\left.[\mathrm{Ma} 1,2.4]\right)$ are splittable. $\mathfrak{E}_{n}(\{2,3\})$ has $n$ atoms, namely 1 ' and $n-1$ atoms below 0 ', such that $\check{a}=a, a ; a=\bar{a}$, and $a ; b=0$ ' whenever $a$ and $b$ are distinct atoms below 0 '. It is not known whether every $\mathfrak{E}_{n}(\{2,3\})$ is representable, but if a given $\mathfrak{E}_{n}(\{2,3\})$ is embeddable in $\mathfrak{R e} U$, then there cannot be three elements $u, v, w \in U$ such that $\langle u, v\rangle,\langle v, w\rangle$, and $\langle u, w\rangle$ are all in the relation correlated with any diversity atom $a$, since $a \cdot a ; a=0$. Therefore, by Ramsey's Theorem, for every $n$ there is some integer $r(n)$ such that if $\mathfrak{E}_{n}(\{2,3\})$ is embeddable in $\mathfrak{R e} U$, then $|U| \leq r(n)$. Thus $r(n)$ is also a bound on the cardinality of the underlying set used to represent any relation algebra obtained from $\mathfrak{E}_{n}(\{2,3\})$ by splitting. If $\mathfrak{A}$ is obtained from $\mathfrak{E}_{n}(\{2,3\})$ by splitting one of its atoms into more than $r(n)$ parts, then any representation of $\mathfrak{A}$ must also contain 
more than $r(n)$ elements. This contradiction shows that such an $\mathfrak{A}$ is not representable.

Algebras constructed in this way can be used to prove Monk's theorem, that RA is not finitely axiomatizable [Mo]. For more details on how this can be done, see [Ma2, Theorems 12 and 13]. (In [Ma2], algebras obtained from $\mathbb{E}_{n}(\{2,3\})$ by splitting are further modified so that they are generated by a single atom.) Monk's original proof used Lyndon algebras. The algebras obtained from $\mathfrak{E}_{n}(\{2,3\})$ by splitting are similar to the nonrepresentable cylindric algebras used by Monk to show the representable $C_{\alpha}$ 's are not finitely axiomatizable whenever $3 \leq \alpha<\omega$ (see [HMT, 3.2.76 and 4.1]).

\section{REFERENCES}

[HMT] L. Henkin, J. D. Monk, and A. Tarski, Cylindric algebras, Part II, North-Holland, Amsterdam, 1985.

[J] B. Jónsson, Varieties of relation algebras, Algebra Universalis 15 (1982), 273-298.

[L] R. C. Lyndon, Relation algebras and projective geometries, Michigan Math. J. 8 (1961), 21-28.

[Ma1] R. D. Maddux, Some varieties containing relation algebras, Trans. Amer. Math. Soc. 272 (1982), 501-526.

[Ma2] __ Nonfinite axiomatizability results for cylindric and relation algebras, J. Symbolic Logic 54 (1989), 951-974.

[Mo] J. D. Monk, On representable relation algebras, Michigan Math. J. 11 (1964), 207-210.

(H. Andréka and I. Németi) Mathematical Institute, Budapest PF. 127, H-1364 Hungary

(R. D. Maddux) Department of Mathematics, Iowa State University, Ames, Iowa 50011 